\title{
Bannayan-Riley-Ruvalcaba syndrome
}

INSERM

\section{Source}

INSERM. (1999). Orphanet: an online rare disease and orphan drug data base. Bannayan-

Riley-Ruvalcaba syndrome. ORPHA:109

Bannayan-Riley-Ruvalcaba syndrome (BRRS) is a rare congenital disorder characterized by hamartomatous intestinal polyposis, lipomas, macrocephaly and genital lentiginosis. 